\title{
The ICAR2010 for development of Arabidopsis research beyond 2010
}

\author{
Kazuo Shinozaki
}

Published online: 13 April 2010

(C) The Botanical Society of Japan and Springer 2010

In the past 20 years, Arabidopsis has been used worldwide as the most important model plant to analyze all essential plant functions. In 2000, the Arabidopsis genome sequence was determined with high reliability by the Arabidopsis Genome Initiative (AGI) groups (Arabidopsis Genome Initiative 2000). This Arabidopsis genome sequence has provided fundamental knowledge for plant scientists to analyze genomic information related to all essential functions. After the determination of the Arabidopsis genome sequence in 2000, the Arabidopsis 2010 project, an international cooperative effort, started analyzing all the coding and noncoding genes and their regulatory networks based on genomics and mutant resources. In the United States, the National Science Foundation (NSF) has supported this large project for 10 years, which has been quite successful in promoting not only Arabidopsis research but also plant and crop sciences (see http://www.arabidopsis.org/portals/ masc/projects.jsp). Similar functional genomics projects also started in Europe, Japan, and other parts of the world after 2000. In the international 2010 project, various genomic and mutant resources have been developed and deposited in resource centers. ABRC (http://www.biosci. ohio-state.edu/ plantbio/Facilities/abrc/abrchome.htm) and NASC (http://arabidopsis.info/) are major Arabidopsis resource centers established in 1991 in the United States

This article is published on behalf of the Organizing Committee of the 21st International Conference on Arabidopsis Research (ICAR2010) at Yokohama, Japan, June 6-10, 2010.

http://arabidopsis2010.psc.riken.jp/index.html.

K. Shinozaki $(\square)$

RIKEN Plant Science Center,

1-7-14 Suehiro-Cho, Tsurumi-ku,

Yokohama 230-0045, Japan

e-mail: sinozaki@rtc.riken.jp and Europe, respectively. The Arabidopsis Information Resource (TAIR) is an important portal site for genome information, various databases, resources, and other useful information for Arabidopsis researchers (http://www. arabidopsis.org/). Most plant scientists will agree that the year 2000 was a turning point in the direction of plant research during these past 10 years. For that reason, 2010 is a year of commemoration and celebration for the success of the Arabidopsis 2010 project in the next stage of plant science and crop biotechnology.

We are pleased to host the 21st International Conference on Arabidopsis Research (ICAR2010) at Yokohama, Japan, June 6-10, 2010 (http://arabidopsis2010.psc.riken. jp/index.html). The ICAR is the annual international conference on Arabidopsis thaliana, in which participants present and discuss their latest research results obtained mainly with Arabidopsis. Approximately 800 scientists are expected to participate in the ICAR2010. Because this is the final year of the 2010 project, there will be many excellent presentations and posters reporting the fruitful results of the project.

An important message of the ICAR2010 is "2010 and beyond: Arabidopsis as basic knowledge for the understanding of whole plant functions and translational research to solve global problems." The following points are noteworthy:

- 2010 is a commemorative year for the international Arabidopsis project.

- Arabidopsis research will continue to lead plant science and crop biotechnology from basic knowledge beyond 2010.

- Asian countries will be more active in the promotion of plant science for application to agriculture and biotechnology. 
- International partnership will be more important in contributing to solutions of global problems in food, energy, health, and the environment.

Japanese plant scientists have contributed greatly not only to the genome sequence project but also to the functional genomics project in Arabidopsis. The Kazusa DNA Research Institute played an important role in the Arabidopsis genome sequence project. RIKEN has been carrying out an Arabidopsis functional genomics project at the Yokohama Institute to collect full-length cDNAs and tagged mutants (http://rarge.psc.riken.jp/). In 2001, RIKEN established the BioResource Center at the Tsukuba Institute to open the genomic and mutant resources developed at RIKEN and other Japanese institutes (http://www.brc.riken.go. jp/lab/epd/Eng/). In Japan, the National BioResource Project (NBRP) began collecting, preserving, and distributing biological resources, including plants (http://www.nbrp. jp/index.jsp). These biological resources become more and more important in understanding the various functions and biodiversity of living organisms.

Plant biology in Japan has developed significantly in these past 10 years because of good funding not only for
Arabidopsis and rice genomics but also for special research projects on plant development, environmental responses, metabolism, and other important topics. Science Map 2006 has revealed that plant science is one of the three most outstanding research fields in the life sciences in Japan (http://www.nistep.go.jp/achiev/abs/eng/rep110e/rep110ae. html).

In this special issue of the Journal of Plant Research (JPR) seven representative researchers present interesting articles as milestones marking the significant advance of Arabidopsis research in the past 10 years. We hope that JPR readers will enjoy the articles as well as the ICAR2010 at Yokohama. We can expect further advances in basic plant biology based on knowledge of Arabidopsis and its application to crops and trees in the next decade.

\section{Reference}

Arabidopsis Genome Initiative (2000) Analysis of the genome sequence of the flowering plant Arabidopsis thaliana. Nature $14: 796-815$ 